\title{
VALIDATION OF THE COMPUTATION OF ROCKET NOZZLE ADMITTANCES WITH LINEARIZED EULER EQUATIONS
}

\author{
R. Kathan ${ }^{1}$, D. Morgenweck ${ }^{1}$, R. Kaess ${ }^{2}$, \\ and T. Sattelmayer ${ }^{1}$ \\ ${ }^{1}$ Lehrstuhl fúr Thermodynamik, Technische Universität München \\ 15 Boltzmannstraße, Garching 85748, Germany \\ ${ }^{2}$ Astrium GmbH, Space Transportation, TP 24 \\ München 81663, Germany
}

High pressure fluctuations coupled with unsteady heat release can affect a rocket engine seriously. Especially when the oscillations match eigenmodes such as T1, T1L1 and T2, T2L1, the acoustic pressure amplitude can reach a critical level. This paper deals with the investigation of the nozzle admittance, which is an important value to characterize the influence of the nozzle on the pressure inside the combustion chamber. Two different nozzle geometries are investigated experimentally at high frequencies. A method to decouple the acoustic modes is presented. The results are compared against an existing theory and simulated data.

\section{NOMENCLATURE}

\section{Latin Symbols}

$c \quad$ speed of sound, $\mathrm{m} / \mathrm{s}$

$D$ diameter, $\mathrm{m}$

$f$ frequency, $1 / \mathrm{s}$

$F$ downstream wave, $\mathrm{m} / \mathrm{s}$

$G$ upstream wave, $\mathrm{m} / \mathrm{s}$

$k \quad$ wave number, $1 / \mathrm{m}$

$L$ length, m

$m$ mth transverse mode

$\dot{m}$ massflow, $\mathrm{kg} / \mathrm{s}$

$n \quad n$th root of $\partial J_{m} / \partial r$

$p$ pressure, $\mathrm{Pa}$

$r$ radial coordinate, $\mathrm{m}$

$R$ radius, $\mathrm{m}$ 


$\begin{array}{ll}s & \text { root of } \partial J_{m} / \partial r \\ t & \text { time, } \mathrm{s} \\ u & \text { axial velocity, } \mathrm{m} \\ x & \text { axial coordinate, } \mathrm{m} \\ Y & \text { admittance, } \mathrm{m}^{2} \mathrm{~s} / \mathrm{kg} \\ \mathscr{Y}=Y \bar{\rho} \bar{c} & \text { nondimensional admittance }\end{array}$

\section{Greek Symbols}

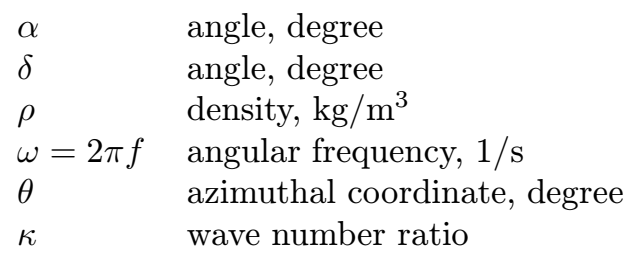

\section{Head pointer}

$$
\begin{array}{ll}
\bar{a} & \text { mean value } \\
\hat{a} & \text { complex amplitude } \\
a^{\prime} & \text { fluctuation } \\
\vec{a} & \text { vector }
\end{array}
$$

\section{Indices}

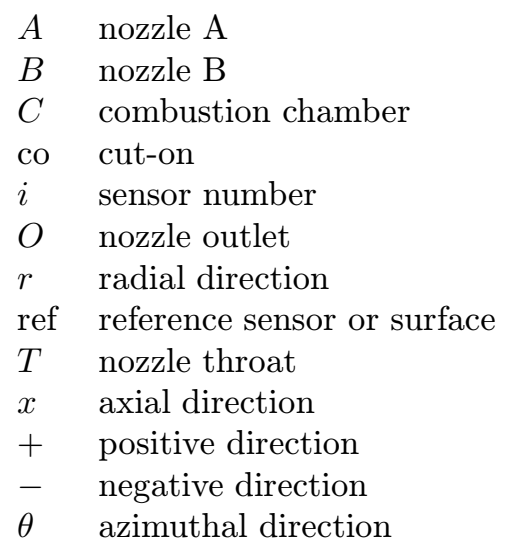

\section{Superscripted Symbols}

0 mode class TOLR0

1 mode class T1LR0

2 mode class T2LR0 


\section{Mathematic Operators and Functions}

$\begin{array}{ll}i\left(i^{2}=-1\right) & \text { imaginary unit } \\ \mathfrak{I} & \text { imaginary part } \\ J_{m} & \text { Bessel function of the first kind of order } m \\ \mathfrak{R} & \text { real part } \\ \nabla\left(\frac{\partial}{\partial x}, \frac{\partial}{\partial y}, \frac{\partial}{\partial z}\right) & \text { differential operator }\end{array}$

\section{Nondimensional Numbers}

$$
\mathrm{M}=u / c \quad \text { Mach number }
$$

\section{INTRODUCTION}

One of the major challenges in the development of rocket engines is the prediction of combustion instabilities. They are a result of high-pressure fluctuations coupled with unsteady heat release [1]. Under certain conditions, this phenomenon can affect the combustion chamber tremendously, resulting in a complete failure of the engine. Consequently, the acoustic pressure fluctuations inside the combustion chamber have to be known. Especially when high frequency oscillations match higher eigenmodes such as T1, T1L1 and T2, T2L1, the pressure amplitude can reach a critical level and affect the heat release. Therefore, these modes have to be considered. Beside the combustion chamber, liners, and faceplate, the main component that affects the pressure inside the engine is the nozzle.

The Lehrstuhl für Thermodynamik at the Technische Universität München performs research activities in the field of acoustics. Different test facilities and numerical tools are used for the investigations of rocket engines and stationary gas turbines with the aim of getting a better understanding of the physical processes yielding to combustion instabilities. The focus is on the investigation of new design rules for future engines in terms of stability predictions. One of the main fields of the experimental research is the validation of numerical simulations and the allocation of adequate boundary conditions. The admittance is an appropriate parameter to describe the acoustics of a passive element such as a nozzle. Since the wave propagation is known for duct elements like a cylindrical combustion chamber, the admittance can be calculated with the impedance tube method. Previous investigations showed a dependency of the admittance on the frequency and the shape of the corresponding eigenmodes. The admittance is a qualitative value for stability predictions and can be transformed into a reflection coefficient. Therefore, it has a major influence on acoustic losses [2]. A negative real part of the admittance can destabilize the rocket engine [3-5]. 
To validate numerically computed nozzle admittances, the measurements were undertaken. The presented test facility is operated under cold conditions with pressurized air and consists of a nozzle and a cylindrical combustion chamber. It can be excited at specific frequencies by a siren. Two different types of nozzles are used. The first nozzle (A) represents a scaled geometry of a real engine. The second one (B) represents a generic geometry of an engine design that is used to conserve the flow field characteristics of the real engine. Both nozzles are photoscaled to fit the chamber diameter of the testrig of $D_{C}=92 \mathrm{~mm}$ which leads to different nozzle throat diameters. The chamber is equipped with sensors in axial and circumferential directions to provide dynamic pressure signals. The one-dimensional (1D) impedance tube method considers the homogeneous axial mean flow in the chamber and provides the nozzle admittance for different modes. It is extended to consider the first $(\mathrm{T} 1, \mathrm{~T} 1 \mathrm{~L} 1, \ldots)$ and the second transverse $(\mathrm{T} 2, \mathrm{~T} 2 \mathrm{~L} 1, \ldots)$ modes.

The experimental results are used to validate the 1D Linearized Euler Equation (LEE) tool provided by Zinn et al. [4]. Furthermore, the results are compared with the three-dimensional (3D) LEE tool PIANO-SAT [5].

\section{THEORY}

Rocket chambers usually consist of a cylinder and an attached nozzle. Since the length and the diameter of the cylinder are very often of similar size, a purely 1D approach is improper. Instead, the $3 \mathrm{D}$ wave field has to be considered.

The pressure and the axial velocity can be written as the sum of mean and fluctuation values:

$$
\begin{aligned}
& p=\bar{p}+p^{\prime} ; \\
& u=\bar{u}+u^{\prime} .
\end{aligned}
$$

Considering only harmonic oscillations, the Helmholtz equation [6] can be found:

$$
-\frac{\omega^{2}}{c^{2}} \Psi_{k}-\nabla^{2} \Psi_{k}=0
$$

with pressure fluctuations rewritten as an infinite sum of normal modes [6]:

$$
p^{\prime}=\sum_{k=1}^{\infty} \hat{p}_{k} \exp (i \omega t) \Psi_{k} .
$$

A solution for typical rocket geometries can be found by a separation approach and a transformation into cylindrical coordinates:

$$
\Psi_{k}=X_{k}(x) R_{k}(r) \Theta_{k}(\theta) .
$$


Table 1 Characteristic functions and wave numbers

\begin{tabular}{|c|c|c|}
\hline Direction & Function & Wave number \\
\hline Radial & $R_{k}=\mathrm{J}_{m}\left(k_{r}^{m n} r\right)$ & $k_{r}^{m n}=\frac{s^{m n}}{R}$ \\
\hline Transverse & $\Theta_{k} \cong \cos \left(k_{\theta}^{m n} \theta+\delta^{m}\right)$ & $k_{\theta}^{m n}=m$ \\
\hline Axial & $\begin{aligned} X_{k}= & \bar{\rho} \bar{c}\left[F^{m n} \exp \left(-i k_{x+}^{m n} x\right)\right. \\
& \left.+G^{m n} \exp \left(-i k_{x-}^{m n} x\right)\right]\end{aligned}$ & $\begin{array}{l}k_{x+}^{m n}=\frac{k}{1-M^{2}} \\
\times\left(-\mathrm{M}+\sqrt{1-\left(\frac{k_{r}^{m n}}{k}\right)^{2}\left(1-\mathrm{M}^{2}\right)}\right) \\
k_{x-}^{m n}=\frac{k}{1-\mathrm{M}^{2}} \\
\times\left(-\mathrm{M}-\sqrt{1-\left(\frac{k_{r}^{m n}}{k}\right)^{2}\left(1-\mathrm{M}^{2}\right)}\right.\end{array}$ \\
\hline
\end{tabular}

Table 2 Cut-on frequencies for different modes

\begin{tabular}{lccccccc}
\hline \multirow{2}{*}{ Mode class } & $m$ & $n$ & \multirow{2}{*}{ Form } & \multirow{2}{*}{$s^{m n}$} & \multicolumn{2}{c}{ Cut-on frequencies, Hz } \\
\cline { 6 - 8 } & & & & & Nozzle A & Nozzle B \\
\hline Longitudinal & 0 & 0 & 0 & L1, L2, $\ldots$ & 0 & - & - \\
1. Radial & $0 \mathrm{R} 1$ & 0 & 1 & $\mathrm{R} 1, \mathrm{R} 1 \mathrm{~L} 1, \mathrm{R} 1 \mathrm{~T} 1, \ldots$ & 3.8318 & 4322 & 4306 \\
2. Radial & $0 \mathrm{R} 2$ & 0 & 2 & $\mathrm{R} 2, \mathrm{R} 2 \mathrm{~L} 1, \mathrm{R} 2 \mathrm{~T} 1, \ldots$ & 7.0155 & 7914 & 7883 \\
1. Transverse & 1 & 1 & 1 & $\mathrm{~T} 1, \mathrm{~T} 1 \mathrm{~L} 1, \mathrm{~T} 1 \mathrm{R} 1, \ldots$ & 1.8412 & 2077 & 2069 \\
2. Transverse & 2 & 2 & 1 & $\mathrm{~T} 2, \mathrm{~T} 2 \mathrm{~L} 1, \mathrm{~T} 2 \mathrm{R} 1, \ldots$ & 3.0541 & 3445 & 3432 \\
\hline
\end{tabular}

These three functions describe the mode shape in the appropriate direction. The solutions in radial and transverse directions for a hard cylinder wall can be easily found in the literature [7]. In axial direction, the mean flow is considered in the up- and downstream wavenumbers. By introducing an up- $(G)$ and downstream $(F)$ waves, the axial solution can be found.

Table 1 gives an overview on the functions and the corresponding wave numbers as a function of the principal wave number $k=\omega / c$. Due to hardware limits, the highest excitation frequency for nozzle A was set to $4000 \mathrm{~Hz}$ and to $3000 \mathrm{~Hz}$ for nozzle B. Considering these frequencies, the modes with cut-on frequencies* $^{*} 8$ ] above these values cannot propagate ${ }^{\dagger}$. Therefore, pure longitudinal (T0LR0), the first (T1LR0), and the second (T2LR0) transverse mode classes are considered. Table 2 gives an overview on the cut-on frequencies for frequencies below $4306 \mathrm{~Hz}$. It is more convenient to neglect the superscripted index $n$

* The notation cut-off frequency can also be found in the literature.

${ }^{\dagger}$ For $f<f_{\text {co }}$, the axial wave number $k_{x \pm}^{n m}$ is complex which leads to damping. 
since it is always equal to zero or one in this case. The complex pressure and velocity amplitude can be written into

$$
\begin{aligned}
& \hat{p}=\hat{p}^{0}+\hat{p}^{1}+\hat{p}^{2} \\
& \hat{u}=\hat{u}^{0}+\hat{u}^{1}+\hat{u}^{2}
\end{aligned}
$$

with the following expressions at the cylinder surface $\left(r=R_{C}\right)$ :

$$
\begin{aligned}
& \hat{p}^{0}=\bar{\rho} \bar{c}\left[F^{0} \exp \left(-i k_{x+}^{0} x\right)+G^{0} \exp \left(-i k_{x-}^{0} x\right)\right] J_{0}\left(s^{0}\right) ; \\
& \hat{p}^{1}=\bar{\rho} \bar{c}\left[F^{1} \exp \left(-i k_{x+}^{1} x\right)+G^{1} \exp \left(-i k_{x-}^{1} x\right)\right] J_{1}\left(s^{1}\right) \cos \left(1 \theta+\delta^{1}\right) ; \\
& \hat{p}^{2}=\bar{\rho} \bar{c}\left[F^{2} \exp \left(-i k_{x+}^{2} x\right)+G^{2} \exp \left(-i k_{x-}^{2} x\right)\right] J_{2}\left(s^{2}\right) \cos \left(2 \theta+\delta^{2}\right) ; \\
& \hat{u}^{0}=\left[\kappa_{+}^{0} F^{0} \exp \left(-i k_{x+}^{0} x\right)-\kappa_{-}^{0} G^{0} \exp \left(-i k_{x-}^{0} x\right)\right] J_{0}\left(s^{0}\right) ; \\
& \hat{u}^{1}=\left[\kappa_{+}^{1} F^{1} \exp \left(-i k_{x+}^{1} x\right)-\kappa_{-}^{1} G^{1} \exp \left(-i k_{x-}^{1} x\right)\right] J_{1}\left(s^{1}\right) \cos \left(1 \theta+\delta^{1}\right) ; \\
& \hat{u}^{2}=\left[\kappa_{+}^{2} F^{2} \exp \left(-i k_{x+}^{2} x\right)-\kappa_{-}^{2} G^{2} \exp \left(-i k_{x-}^{2} x\right)\right] J_{2}\left(s^{2}\right) \cos \left(2 \theta+\delta^{2}\right)
\end{aligned}
$$

where $\kappa_{ \pm}^{m}=k_{x \pm}^{m} /\left(k-\mathrm{M} k_{x \pm}^{m}\right)$ represents a correction factor to achieve the axial velocity component. To compute the pressure and velocity, the up- $\left(F^{m}\right)$ and downstream $\left(G^{m}\right)$ waves and the correction angles $\left(\delta^{m}\right)$ have to be known. This can be achieved by solving the linear equations*:

$$
\hat{p}_{i}-\left[\hat{p}^{0}+\hat{p}^{1}+\hat{p}^{2}\right] \rightarrow \min
$$

where $\hat{p}_{i}$ are the measured pressure values. Since one pressure sensor provides one complex value (or amplitude and phase), at least eight sensors are necessary. Finally, the admittance can be calculated at any position inside the cylindrical combustion chamber for each mode class:

$$
Y^{m}=\frac{\hat{u}^{m}}{\hat{p}^{m}} .
$$

\section{EXPERIMENTAL SETUP}

The measurements were performed at the Lehrstuhl fúr Thermodynamik. The experiment consisted of a cylindrical part and an attached nozzle. Two different nozzles were used in this investigation. Instead of an injector faceplate, a perforated plate was used to homogenize the incoming flow. The experiment was operated with pressurized air without combustion. The air was provided by a high-pressure feed system with several mass flow control units. An overview of

\footnotetext{
${ }^{*}$ The linear equation system was solved with a Levenberg-Marquardt algorithm.
} 


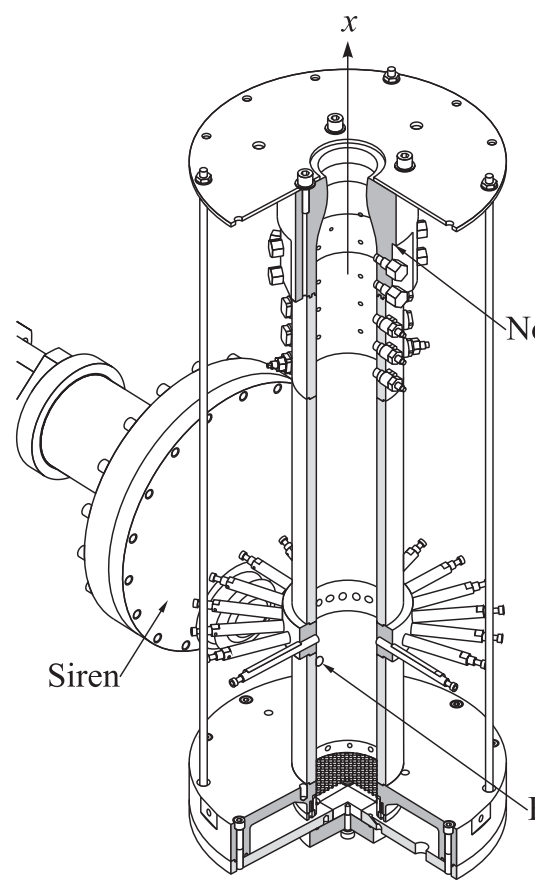

(a)

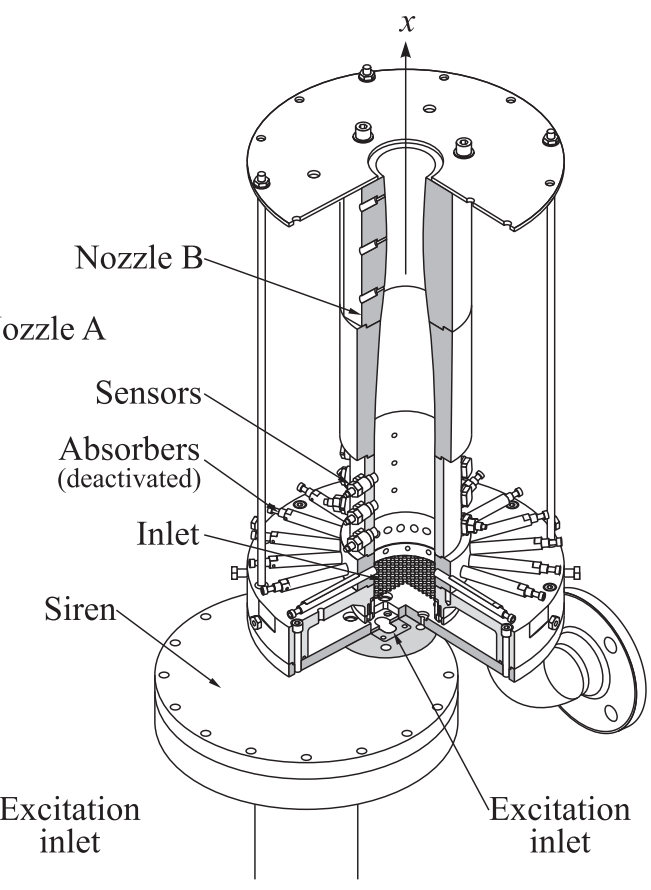

(b)

Figure 1 Nozzles A $(a)$ and B (b)

the test facility in the two different configurations is given in Fig. 1. The geometries of the nozzles have been selected such that they are fully specified using the few geometry parameters of the nozzle available in the admittance calculation code developed by Zinn et al. [4].

The nozzle geometry is shown in Fig. 2 with the appropriate values provided in Table 3. Both nozzles consist of a subsonic and a supersonic part to make sure that the flow is accelerated through the nozzle throat from subsonic to supersonic velocities to ensure sonic conditions in the nozzle throat. An external siren excites the chamber at specific frequencies and is connected sidewise (nozzle A) and coaxial (nozzle B) to the cylindrical combustion chamber. The shape of the siren outlet is adapted such that it provides almost perfect smooth sinusoidal pulses.

The cylindrical combustion chamber has several ports in axial as well as in circumferential directions and can be equipped with dynamic pressure sensors*.

*PCB Piezotronics, model M106B: internally protected against mechanical vibrations, uses a high pass filter to suppress the mean pressure. 


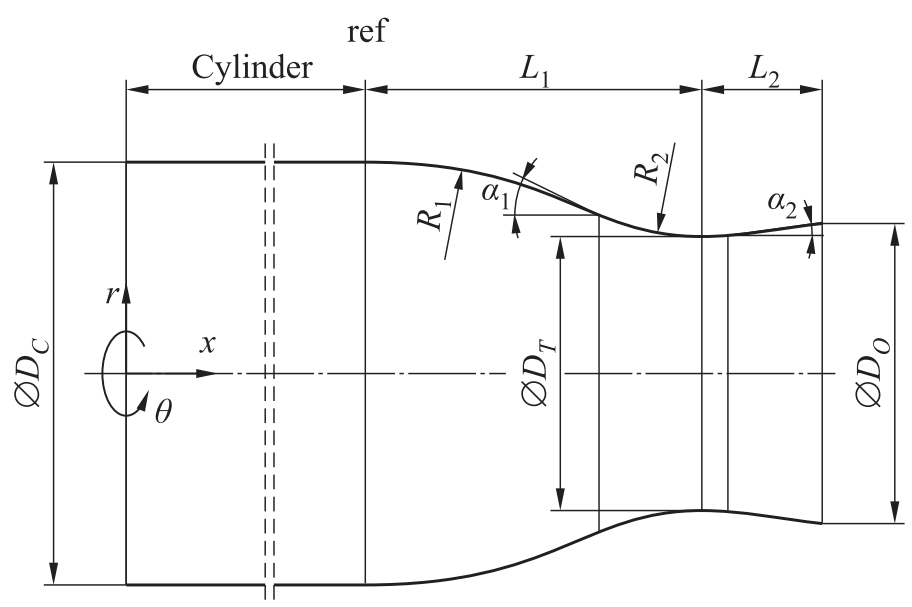

Figure 2 Nozzle contour

Table 3 Parameters of nozzles A and B

\begin{tabular}{c|cc|c}
\hline Symbol & Nozzle A & Nozzle B & Unit \\
\hline$\varnothing D_{C}$ & 92 & 92 & $\mathrm{~mm}$ \\
$\varnothing D_{T}$ & 60 & 56 & $\mathrm{~mm}$ \\
$\varnothing D_{O}$ & 64 & 67 & $\mathrm{~mm}$ \\
$R_{1}$ & 119 & 1469 & $\mathrm{~mm}$ \\
$R_{2}$ & 53 & 544 & $\mathrm{~mm}$ \\
$L_{1}$ & 73 & 273 & $\mathrm{~mm}$ \\
$L_{2}$ & 25 & 81 & $\mathrm{~mm}$ \\
$\alpha_{1}$ & 25 & 7 & degree \\
$\alpha_{2}$ & 6 & 6 & degree \\
\hline$p$ & 1.654 & 1.664 & $\mathrm{bar}$ \\
$\dot{m}$ & 1.150 & 1.000 & $\mathrm{~kg} / \mathrm{s}$ \\
$\mathrm{M}$ & 0.25 & 0.22 & - \\
\hline
\end{tabular}

Thirty two sensors were used in the configuration of nozzle A while their quantity was reduced to 13 for nozzle B. The analog signals were simultaneously sampled* at $2^{15} \mathrm{~Hz}$ for $1 \mathrm{~s}$ of measurement time. Afterwards, they were Fourier transformed to provide amplitude and phase at the corresponding excitation frequency. For each excitation frequency, several recordings were acquired to obtain a representative time average of the acoustic pressure in amplitude and phase.

\footnotetext{
*A/D data acquisition unit: National Instruments NI PXI-4472.
} 


\section{AVAILABLE DATA}

The experimental results are compared by two available sets of data:

- simulated results by Kaess et al. [9]; and

- numerical solution provided by Zinn et al. [4].

\subsection{Simulated Results}

The admittance of the nozzle was computed with the 3D simulation tool PIANOSAT $[1,9,10]$ based on the LEE. The available admittance data are simulated for a frequency close to the cut-on frequency of mode class 1 for nozzle A.

\subsection{Numerical Solution}

Zinn et al. [4] provide numerical results in comparison to the theory developed by Crocco and Sirignano [6] for the admittances of choked nozzles that considers $3 \mathrm{D}$ flow oscillations including higher modes. By specifying the mode class, this method provides the corresponding admittance.

\section{RESULTS}

The experiments presented in this section have been performed for a frequency range from 1000 to $4000 \mathrm{~Hz}$ (nozzle A) and $3000 \mathrm{~Hz}$ (nozzle B) with a step size of $10 \mathrm{~Hz}$. Figure 3 shows the pressure amplitude of one typical sensor as a function of the excitation frequency for both configurations. Below the cut-on frequency of mode class 1 , the values are very small. It can be recognized that the amplitudes at the calculated cut-on frequency which is valid for a pure T1 mode are low as well. Therefore, a pure T1 mode cannot propagate in both investigated configurations. The reason for that is that the up- and downstream boundaries of the cylindrical combustion chamber do not behave like a solid wall and, hence, induce an axial pressure gradient which changes the mode shape into a T1L1 mode. Short above the theoretical cut-on frequency, the amplitude increases by a factor of 20 for nozzle A and 10 for nozzle B.

First, the quality of the reconstructed values is analyzed. Figure 4 shows the pressure measured by two different sensors for the two configurations compared to the reconstructed values following Eq. (1). They are in nearly perfect agreement with each other over the whole frequency range. 


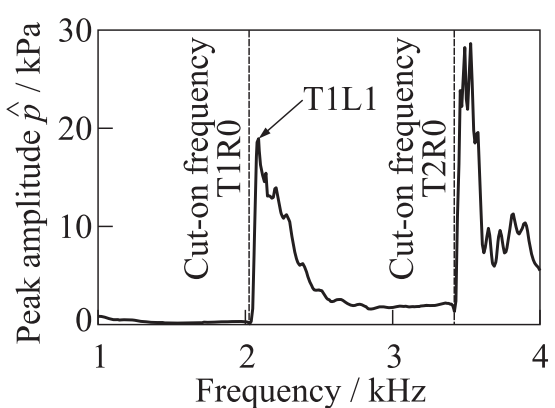

(a)

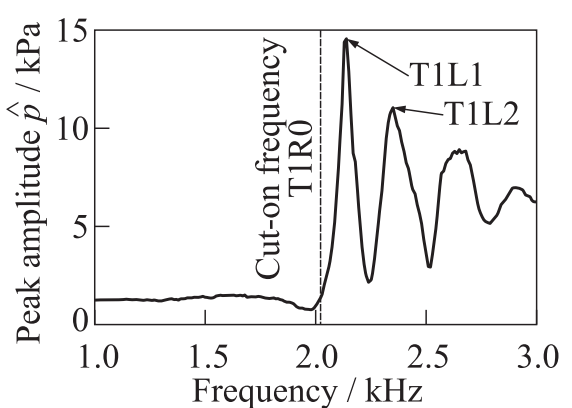

(b)

Figure 3 Pressure amplitudes of one sensor: $(a)$ nozzle A and (b) nozzle B
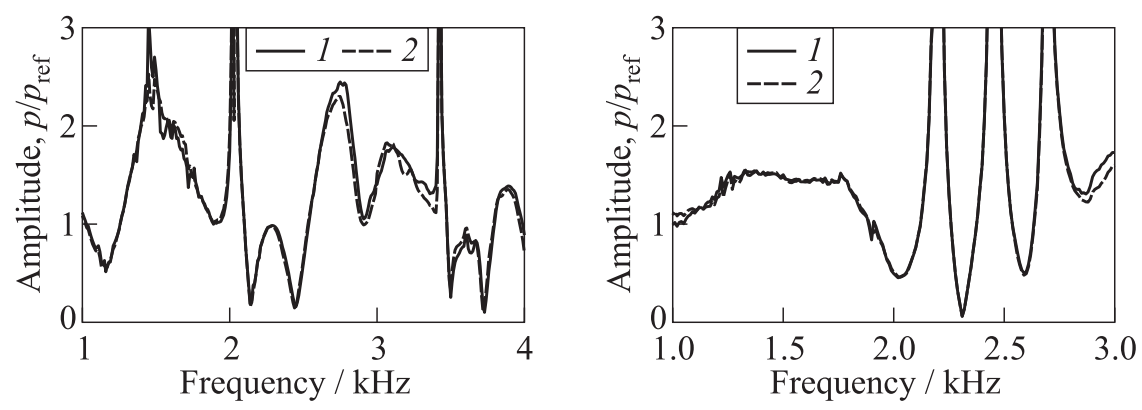

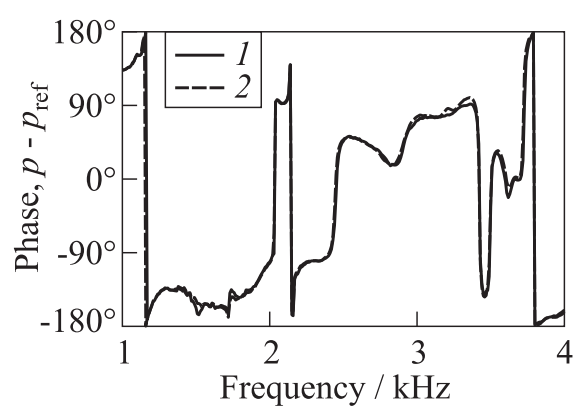

(a)

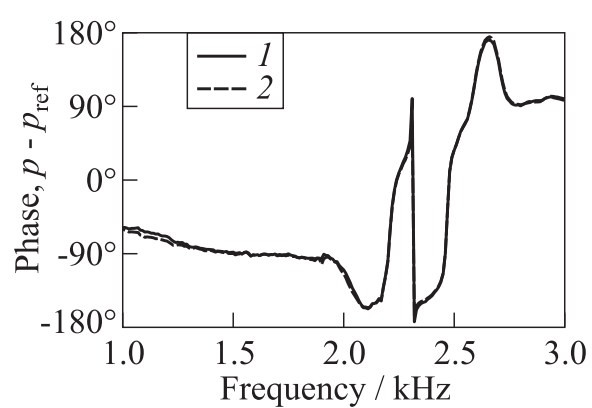

(b)

Figure 4 Measured (1) and reconstructed (2) pressure data of one sensor, considering mode classes 0 and 1, referenced at each frequency to another sensor: (a) nozzle A and (b) nozzle B 
Figures 5 and 6 show the measured admittance compared to the results provided by the theory from Zinn et al. for mode classes 0,1 , and 2 in terms of real and imaginary parts and the simulated results for mode class 1 . It can be recognized that the values of mode classes 0 and 1 match quite well below the cut-on frequency of the next higher mode class. Above, some devi-
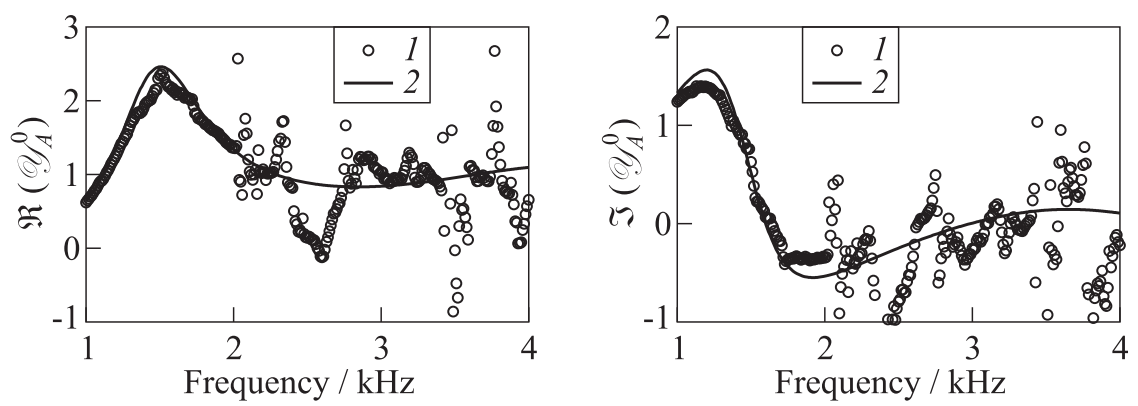

(a)
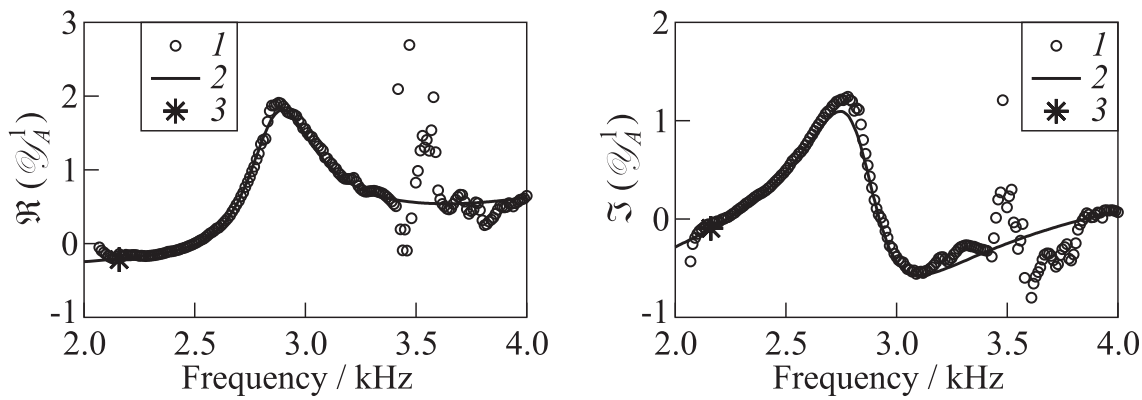

(b)
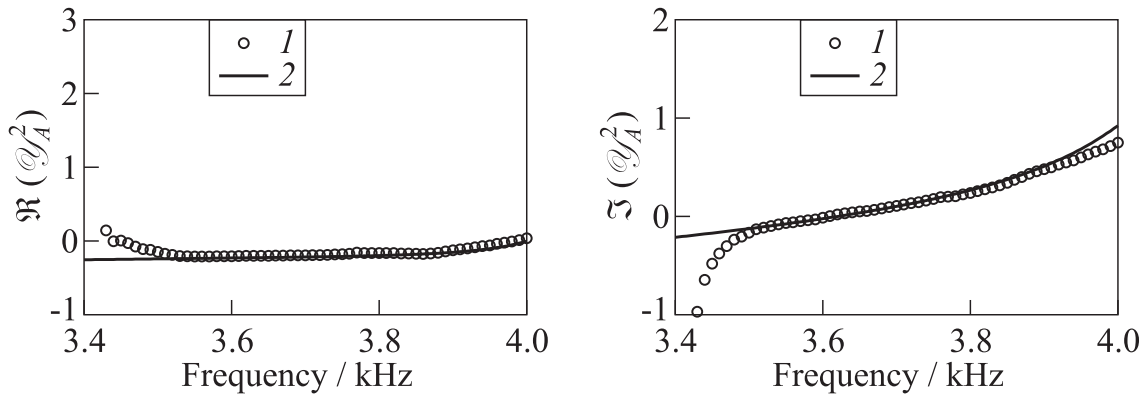

(c)

Figure 5 Admittances of nozzle A (mode classes $0(a), 1(b)$, and $2(c)$ ): 1 experiments; 2 - [4]; and 3 - simulation 


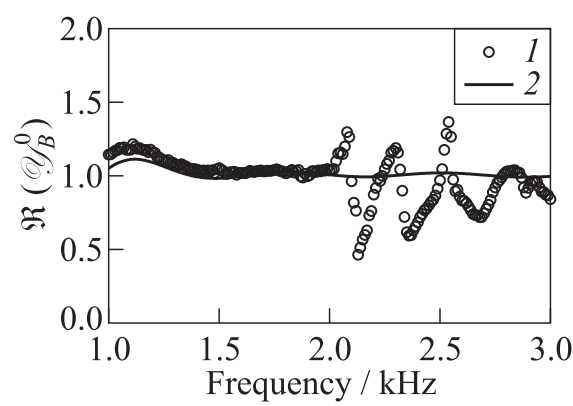

(a)

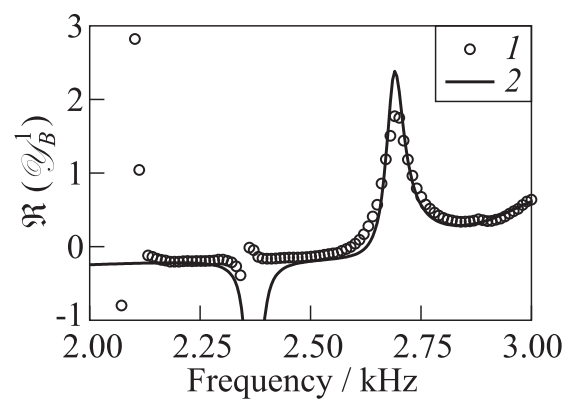

(b)

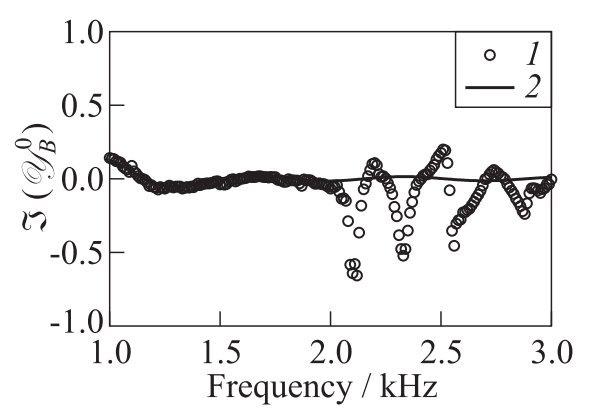

(c)

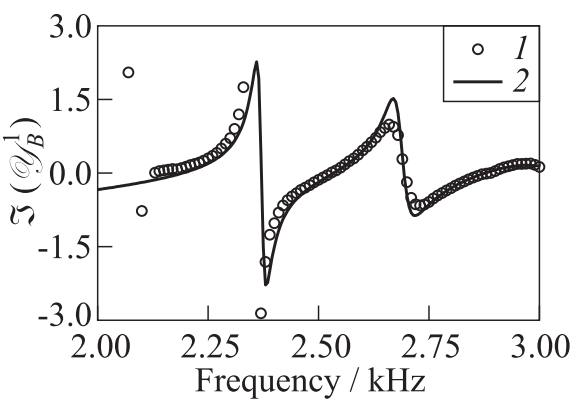

(d)

Figure 6 Admittances of nozzle B (mode classes $0(a)$ and $1(b)$ ): 1 - experiments; and $2-[4]$

ations can be recognized. A reason for that could be that the pressure amplitude of one mode class is very small compared to the one from the next higher mode class (see Fig. 3), assuming the same scale of amplitude short below and above the cut-on frequency of the lower mode class. Since the admittance is the ratio of the velocity fluctuation to the pressure fluctuation, its value can get quite imprecise due to small pressure values in the denominator. Nevertheless, this is irrelevant, because the pressure is dominated by the next higher mode.

Below the theoretical cut-on frequencies, significant deviations can be recognized. They are a result of the fact that mode classes cannot propagate below their cut-on frequency. Hence, these deviations can be neglected. Short above the cut-on frequency, where the pressure amplitudes are high, theory and experiment match quite well. This frequency region is relevant for further stability analysis, since the real part of the admittance is negative and in consequence could change the damping behavior significantly. 


\section{CONCLUDING REMARKS}

The real and imaginary parts of the admittance are in very good agreement with the theoretical values. For all investigated mode classes and nozzles, it can be easily seen that the values of the real and imaginary part of the admittance are equal to the computed values based on the theory provided by Zinn et al. [4] for almost each frequency. At some frequencies, minor differences occur. The simulated results are also in good agreement with the experimental results. For mode class 0 , the absolute values of the real and the imaginary parts of the experimental results are marginally below the theory for the whole frequency range. This yields lower damping. For mode class 1 , it can be concluded that the simulated values and the theory of Zinn et al. can be confirmed. The experiment delivers the same negative real part of the nozzle admittance for specific frequencies which corresponds to a destabilizing effect.

\section{ACKNOWLEDGMENTS}

This project was supported by Astrium GmbH, Space Transportation, and Prof. Dr.-Ing. Jan Delfs from the Institute of Aerodynamics and Flow Technology at the German Aerospace Research Center (DLR) who provided PIANO. Their support is gratefully acknowledged.

\section{REFERENCES}

1. Pieringer, J., T. Sattelmayer, and F. Fassl. 2009. Simulation of combustion instabilities in liquid rocket engines with acoustic perturbation equations. J. Propul. Power 25(5).

2. Morgenweck, D., T. Sattelmayer, F. Fassl, and R. Kaess. 2011. Influence of scaling rules on loss of acoustic energy. J. Spacecraft Rockets 48(3):498-506.

3. Bell, W. A. 1972. Experimental determination of three-dimensional liquid rocket nozzle admittances. Dissertation. Georgia Institute of Technology Atlanta.

4. Zinn, B. T., W. A. Bell, B. R. Daniel, and A. J. Smith, Jr. 1973. Experimental determination of three-dimensional liquid rocket nozzle admittances. AIAA J. 11.

5. Pieringer, J.E. 2008. Simulation Selbsterregter Verbrennungsschwingungen in Raketenschubkammern im Zeitbereich. Dissertation. Technische Universitát München, Institut für Energietechnik, Lehrstuhl für Thermodynamik.

6. Crocco, L., and W. A. Sirignano. 1967. Stability of three-dimensional motions in a combustion chamber. North Atlantic Treaty Organisation. (AGARDograph 117). Forschungsbericht.

7. Morse, P. M., and K. U. Ingard. 1968. Theoretical acoustics. Princeton University Press. 927 p. 
8. Rienstra, S. W., and A. Hirschberg. 2010. An introduction to acoustics. Eindhoven University of Technology.

9. Kaess, R., S. Koeglmeier, M. Schmid, and T. Sattelmayer. 2010. Linearized Euler calculation of acoustics of a rocket combustion chamber. 2nd REST Modelling Workshop Proceedings. Ottobrunn.

10. Delfs, J. W., H.A. Grogger, and T.G.W. Lauke. 2002. Numerical simulation of aeroacoustic noise by DLR's aeroacoustic code PIANO. DLR Braunschweig. Forschungsbericht. 